\title{
The complement system in COVID-19: friend and foe?
}

\author{
Anuja Java, ${ }^{1}$ Anthony J. Apicelli, ${ }^{2}$ M. Kathryn Liszewski, ${ }^{3}$ Ariella Coler-Reilly, ${ }^{3}$ John P. Atkinson, ${ }^{3}$ \\ Alfred H.J. Kim, ${ }^{3}$ and Hrishikesh S. Kulkarni ${ }^{4}$ \\ 'Division of Nephrology, ${ }^{2}$ Department of Radiation Oncology, ${ }^{3}$ Division of Rheumatology, and ${ }^{4}$ Division of Pulmonary and \\ Critical Care Medicine, Department of Medicine, Washington University School of Medicine, Saint Louis, Missouri, USA.
}

\begin{abstract}
Coronavirus disease 2019 (COVID-19), the disease caused by severe acute respiratory syndrome coronavirus-2 (SARS-CoV-2) has resulted in a global pandemic and a disruptive health crisis. COVID-19-related morbidity and mortality have been attributed to an exaggerated immune response. The role of complement activation and its contribution to illness severity is being increasingly recognized. Here, we summarize current knowledge about the interaction of coronaviruses with the complement system. We posit that (a) coronaviruses activate multiple complement pathways; (b) severe COVID-19 clinical features often resemble complementopathies; (c) the combined effects of complement activation, dysregulated neutrophilia, endothelial injury, and hypercoagulability appear to be intertwined to drive the severe features of COVID-19; (d) a subset of patients with COVID-19 may have a genetic predisposition associated with complement dysregulation; and (e) these observations create a basis for clinical trials of complement inhibitors in life-threatening illness.
\end{abstract}

Authorship note: AHIK and HSK are co-senior authors and contributed equally to this work.

Conflict of interest: AJ reports serving on the scientific advisory boards of Alexion Pharmaceuticals and Novartis Pharmaceuticals and serving as a consultant for Cemini Therapeutics. JPA reports serving as a consultant for Celldex Therapeutics, Clinical Pharmacy Services, Kypha Inc., Achillion Pharmaceuticals Inc., and BioMarin Pharmaceutical Inc. and stock or equity options in Compliment Corporation, Kypha Inc., Cemini Therapeutics, and AdMiRx Inc. AHJK reports personal fees $(<\$ 10,000)$ from Exagen Diagnostics Inc. and GlaxoSmithKline.

Copyright: () 2020, American Society for Clinical Investigation.

Reference information: /CI Insight. 2020;5(15):e140711.

https://doi.org/10.1172/jici.

insight.140711.

\section{Introduction}

The emergence of the coronavirus disease 2019 (COVID-19) pandemic, caused by severe acute respiratory syndrome coronavirus-2 (SARS-CoV-2), has resulted in a rapid response from the global scientific community to better understand its pathophysiology (1-3).

Despite knowledge gained from SARS-CoV and Middle East respiratory syndrome-coronavirus (MERS-CoV) outbreaks, many aspects of this pandemic are unique to SARS-CoV-2. For example, COVID-19 has a wide spectrum of presentation, ranging from asymptomatic carriers to severe infections resulting in death $(4,5)$, with $5 \%-25 \%$ of patients requiring ICU-level support $(6,7)$. Critically ill patients suffer from acute respiratory failure, circulatory shock, acute renal failure, and thrombotic complications, including cerebrovascular accident (CVA), myocardial infarction, and pulmonary embolism as well as a hyperinflammatory state (8). Although multiorgan involvement has been the hallmark of severe SARS$\mathrm{CoV}-2$ infection, certain features specific to COVID-19 are being increasingly recognized (8). One such example is the rapid clinical deterioration in cardiorespiratory status, reminiscent of cytokine release syndrome (9-11). Additionally, a subset of those developing COVID-19 have evidence of ongoing clinical and laboratory evidence of ongoing thromboses, confirmed in multiple autopsy reports, with features that overlap with thrombotic microangiopathies (TMAs) (12-14). These reports, in combination with results from both targeted and unbiased proteomic studies, have increased the likelihood that complement activation is a key contributor to the COVID-19 pathogenesis (12-17). In this Review, we summarize interactions between coronaviruses and the complement system, with a specific focus on SARS-CoV-2. We also describe clinical trials that are underway for the treatment of severe COVID-19.

\section{Interplay between SARS-CoV-2 and the complement system}

Antiviral strategies. The complement system traces its origins to more than a billion years ago when primitive proteins evolved to protect cells from pathogens and to engage in intracellular metabolic processes $(18,19)$ (Figure 1A). The contemporary complement system lies at the interface between innate and adaptive immunity (20). It efficiently recognizes and eliminates viral pathogens via several mechanisms: opsonizing viruses and virus-infected cells (including lysing them), inducing an antiviral immunoinflammatory state, boosting virus-specific immune responses, and directly neutralizing cell-free viruses (21) (reviewed in ref. 22). 
Antihost strategies. Viruses seek to disable and exploit complement activation using varied strategies (reviewed in ref. 23). For example, poxviruses attenuate complement activation by expressing a protein with functional and structural homology to complement regulatory proteins (24). Similarly, flaviviruses hijack complement regulators to avoid their engagement and thereby inhibit antiviral activity (reviewed in ref. 23). Certain viruses, such as HIV-1, recruit host complement regulators into their virions (23). In some of these cases, $\mathrm{C} 3$ attaches to the surface of viruses and may be carried intracellularly; however, the ability of viruses to cleave C3 may affect host antiviral responses (25). Whether coronaviruses exhibit such antiviral strategies (i.e., disabling and exploiting the complement system) remains to be answered.

Coronavirus-mediated complement activation. Emerging in vitro and in vivo data indicate that complement activation plays a critical role in pathogenesis and disease severity of SARS-CoV and SARS-CoV-2. Using virus-infected $\mathrm{C}^{-1-}$ mice, Gralinski et al. assessed complement activation in SARS-CoV infection (26). $\mathrm{C} 3$ activation products ( $\mathrm{C} 3 \mathrm{a}, \mathrm{C} 3 \mathrm{~b}, \mathrm{iC} 3 \mathrm{~b}, \mathrm{C} 3 \mathrm{c}$, and $\mathrm{C} 3 \mathrm{dg}$ ) were observed in the lung as early as 1 day after SARS-CoV infection. Lung injury and weight loss were significantly reduced in the absence of C3, despite an unchanged viral load. Further, substantially fewer neutrophils and inflammatory monocytes, as well as lower cytokine and chemokine levels, were detected in C3-deficient mice. Additionally, factor $\mathrm{B}^{-/-}$or $\mathrm{C}^{-/-}$ mice also had less weight loss than WT mice (26), suggesting that complement activation enhances pulmonary pathology and SARS-CoV infection-associated systemic illness. A preprint by Gao et al. reported that the secreted nucleoprotein (N protein) dimers of MERS-CoV, SARS-CoV-1, or SARS-CoV-2 autoactivate mannan-binding lectin-associated serine protease 2 (MASP-2), the primary enzymatic initiator of the lectin pathway (Figure 1B) (27). MASP-2 activation leads to generation of C3 convertase (via cleavage of C4 and $\mathrm{C} 2$ ) and membrane attack complex (MAC) formation. Moreover, alteration of the MASP-2-binding motif, either via Masp2 deletion or blocking the MASP-2-N protein interaction, attenuated lung injury. These data, along with human proteomic studies $(15,16)$, suggest that coronavirus infections involve the activation of multiple complement pathways.

Natural antibodies. Natural antibodies are polyreactive germline-encoded antibodies produced by B-1 cells capable of binding evolutionarily fixed epitopes, such as phospholipids and glycoproteins (28). A role of natural antibodies in COVID-19 has been suggested by observations that both the infection and death rates from COVID-19 in hospitals in China, the United States, Spain, and Italy are skewed toward patients with blood group A and away from those with blood group O (29-31). A similar finding has also been previously published for SARS-CoV-1 (32). As type O patients harbor both anti-A and anti-B natural IgM, these antibodies may help to reduce the viral load of their hosts due to early activation of the classical complement pathway and viral clearance before the establishment of a pneumonia. In fact, such a hypothesis has been tested in vitro, using a measles virus produced in cells engineered to express only A-type, B-type, or O-type carbohydrate epitopes. In studies using human preimmune serum, neutralizing antibodies were present and prevented viral infection by A-type and B-type, but not O-type, virus in a complement-dependent manner (33). Furthermore, in vitro expression of SARS-CoV-1 spike protein (S protein) ectodomain in CHO cells (also engineered to express A-transferase) demonstrated impaired adherence to ACE2-expressing cell lines when either a S protein-specific monoclonal antibody or isolated human natural IgM anti-A antibodies were used (34). Taken together, these data imply that natural ABO group antibodies serve as an initial barrier to viral infection in a complement-dependent manner.

Interestingly, older patients have reduced diversity of natural IgM antibodies (35). As has been widely reported from data sets from China (36), Italy (37), and the United States (38, 39), the case fatality rate of COVID-19 rises with age. It is tempting to speculate that, in the relatively asymptomatic early phases of the disease, reduced natural antibody diversity may lead to poor control of virus clearance. This then predisposes older patients to be more prone to developing severe pneumonia and other complications of disease.

In sum, many intriguing questions remain unanswered and will require further in-depth study. For example, how is SARS-CoV-2 protected from complement-mediated inactivation strategies? Does SARSCoV-2 exploit complement or its receptors to gain entry into cells? Does promotion of complement activation assist viral pathogenesis? Investigating the temporal nature of the host immune response to SARS-CoV (vis-à-vis complement proteins) will likely provide additional insights and facilitate the personalizing of therapeutics to both promote host defense and minimize tissue injury. 
A

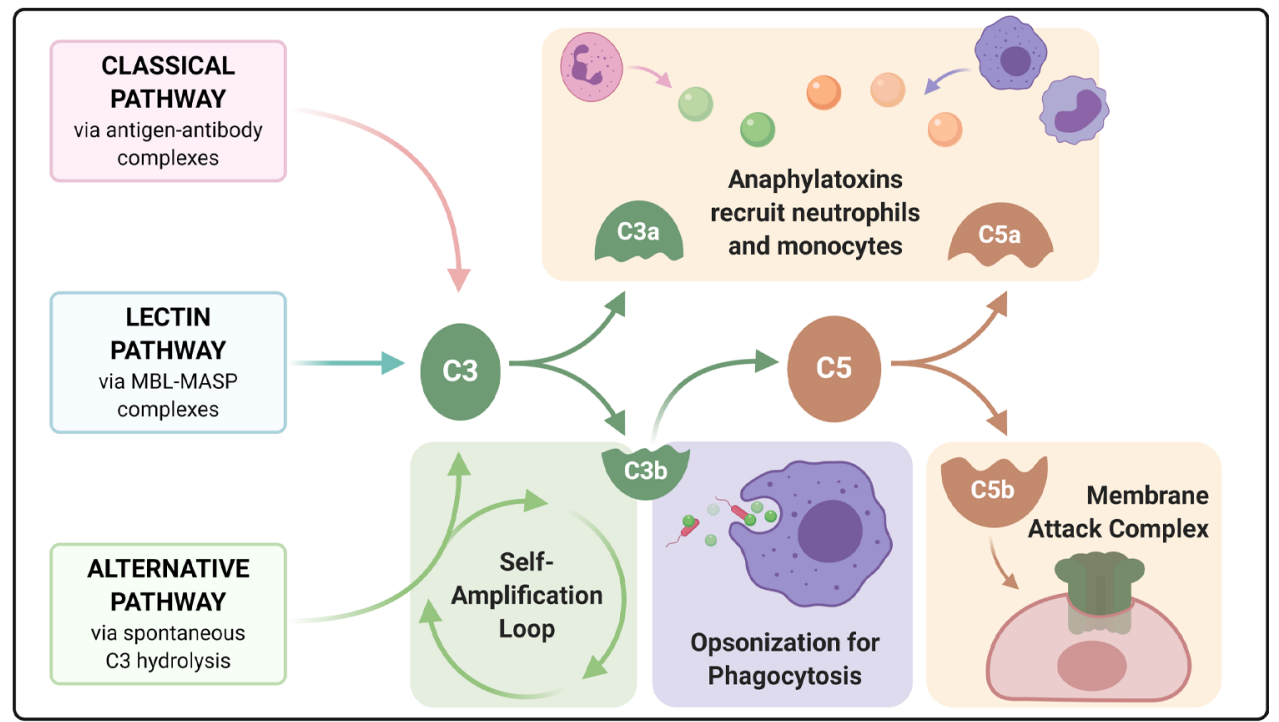

$\mathbf{B}$

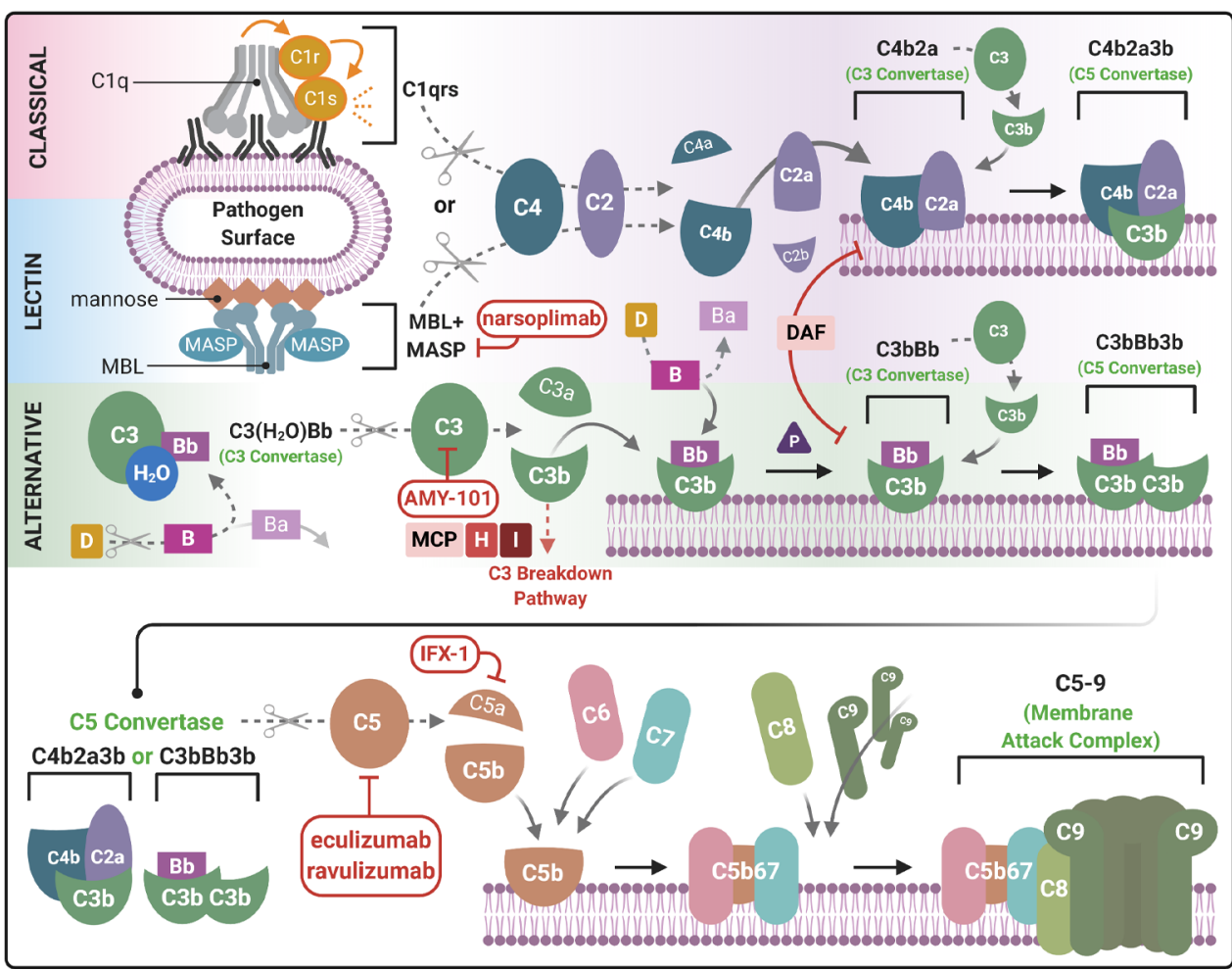

Figure 1. Schematic diagram of the activation of the 3 complement cascades and COVID-19 therapeutically targeted components. A simplified (A) and detailed (B) description of the complement cascade are shown. There are 3 major independent, but overlapping, pathways for activation of complement. The classical, lectin, and alternative pathways generate the major complement opsonin C3b and the membrane attack complex (MAC, C5b-9). The engagement of the $\mathrm{C} 1$ complex (C1qrs) with antigen-antibody complexes or pentraxins generates bimolecular C3 convertase (C4b2a). The latter cleaves C3 to $\mathrm{C} 3 \mathrm{~b}$ and releases the anaphylatoxin, $\mathrm{C3}$ a. The binding of another $\mathrm{C3b}$ to the convertase generates the $\mathrm{C} 5$ convertase (C4b2a3b). The lectin pathway is similar, except mannose-associated serine proteases (MASPs) substitute for C1 proteases (C1r and C1s). The AP self-activates by the low-level turnover of C3 in serum to $C 3\left(\mathrm{H}_{2} \mathrm{O}\right)$. This nondiscriminate tickover, particularly on a pathogen surface or damaged tissue, rapidly engages factors $B(B)$ and $D(D)$ to form a $C 3$ convertase $\left[3\left(\mathrm{H}_{2} \mathrm{O}\right) \mathrm{Bb}\right.$ to trigger more $\mathrm{C3b}$. C3b generated by any one of the 3 pathways opsonizes the target, binds a $C 3$ convertase to generate the trimolecular $\mathrm{C} 5$ convertases (C4b2a3b or C3bBbC3b), and amplifies more C3b via the AP. Cleavage of C5 releases the potent anaphylatoxin C5a, while the C5b initiates the terminal pathway to form the MAC. C3a and C5a are potent chemoattractants for neutrophils and monocytes. Membrane-bound (CD46, CD55) and fluid-phase regulators (factor $\mathrm{H}$ [FH], factor I [FI]) prevent fluid-phase activation and activation on normal cells and tissues. For example, membrane cofactor protein (MCP, also known as CD46) serves as a cofactor the FI-mediated inactivation of C3b to iC3b and C4b to C4c and C4d. The current armamentarium of complement inhibitors being tested in COVID-19 targets different aspects of this cascade. AMY-101 is a synthetic peptide that inhibits C3, whereas both eculizumab and ravulizumab are humanized monoclonal antibodies against C5. IFX-1 is a monoclonal antibody specifically targeting C5a, and narsoplimab is a human monoclonal antibody targeting MASP-2. 


\section{Clinicopathological features of COVID-19 indicative of complement activation}

General features. Soluble markers of complement activation have been observed in patients with COVID-19. In a single-center case series from Italy, increased levels of plasma C5a and sC5b-9 were noted in patients with moderate (patients requiring continuous positive airway pressure) and severe (mechanically ventilated) COVID-19 (40). In addition, complement fragment deposition has been reported in multiple organs in patients with COVID-19. For example, septal capillary injury in the lungs of those who died of respiratory failure is accompanied by extensive deposits of C5b-9, C4d, and MASP-2 in the microvasculature (13). A similar pattern has been demonstrated in the skin of patients with retiform and purpuric lesions (13). Moreover, C4d and C5b-9 colocalized with the SARS-CoV-2 S protein in the lung and skin vasculature (13). At autopsy, strong C5b-9 staining was demonstrated on the apical brush border of tubular epithelial cells of the kidney, with minimal deposition on glomeruli and capillaries (41). Reports have proposed that the relative lack of complement regulators on the proximal tubular cells in the kidney makes them more susceptible to complement-mediated injury $(42,43)$. In further support of this hypothesis, genetic variants in 2 complement regulators, DAF (also known as CD55) and FH, have been associated with SARS-CoV-2 infection (44) and have previously been linked to atypical hemolytic uremic syndrome (aHUS) and age-related macular degeneration (AMD), which are associated with haploinsufficiency of complement regulatory proteins (45). Of interest, AMD was identified as a risk factor for mortality in COVID-19 (44). While these associations need to be evaluated in independent cohorts, they suggest that activation of the lectin and alternative pathways of complement is preferentially occurring in COVID-19.

There are certain clinicopathologic features of COVID-19 that are distinct from the described complementopathies. In contrast to the relatively limited number of organs affected in AMD and aHUS (which is a renal-predominant TMA, although extrarenal manifestations have been described), COVID-19 has a wider spectrum of organ involvement. This may be a result of the broad-organ tropism of SARS-CoV-2 (46) and resultant damage induced by both the virus and the immune response to it, which is especially true in the lung. Additionally, evidence of hemolysis has not been observed in COVID-19 (47).

Endotheliopathy. Histopathologic features in COVID-19 suggest a complement-mediated endotheliopathy. Endothelial cell abnormalities have been observed in numerous organs, including the kidney, lung, heart, small bowel, and liver $(48,49)$. Endothelial cell swelling with variable foamy degeneration was present in the glomeruli of 5 of 26 deceased patients with COVID-19 (50). In 3 additional patients, segmental fibrin thrombi in glomerular capillary loops were identified and associated with severe injury to the endothelium (50). Patients with COVID-19 have also been found to have biopsy-proven TMA (ref. 51 and A. Chang, personal communication). These cytomorphologic changes in the endothelium are prototypic of complement-mediated injury induced by C5b-9 (52). Indeed, endothelial C5b-9 deposition in patients with COVID-19 has been observed by Magro et al. (13). Given the prevalence of ACE2 expression on vascular endothelium, it is hypothesized that the endothelium is infected by SARS-CoV-2 (49), which induces injury, activates complement, and sets up a feedback loop that perpetuates inflammation (53). In support of this hypothesis, substantially elevated levels of vWF have been identified in patients with severe SARS CoV-2 infection, consistent with endothelial injury $(54,55)$. Hypertension, diabetes, obesity, and established cardiovascular disease have also been identified as independent COVID-19 risk factors, indicating that preexisting conditions associated with endothelial dysfunction may heighten risk of both susceptibility and adverse outcomes in this illness (38). Additionally, other triggers specific to COVID-19 that could lead to endotheliopathy include neutrophilic extracellular traps (NETs) (56) and hypoxia (57).

Hypercoagulable state. A hypercoagulable state distinct from disseminated intravascular coagulation (DIC) is superimposed upon and likely contributes to the endothelial injury and complement activation in severe SARS-CoV-2 infection (55). Complement activation has been described in severe cases, and an admission D-dimer level $>1 \mu \mathrm{g} / \mathrm{L}$ predicts an 18-fold increase of in-hospital mortality (58). However, distinguishing features of hypercoagulability in COVID-19 include a normal prothrombin time (PT) but activated partial thromboplastin time (aPTT), an elevated fibrinogen level, a normal or increased platelet count, near-normal antithrombin level, and increased protein C and factor VIII levels (55).

Although most patients with COVID-19-associated acute respiratory distress syndrome (ARDS) present with varying degrees of diffuse alveolar damage, a subset of patients have increased dead-space ventilation with high compliance, suggestive of pulmonary microthrombi $(13,59,60)$. Autopsy studies in some patients with COVID-19 have demonstrated a septal microangiopathy characterized by endothelial cell injury, mural fibrin deposition, and variable intraluminal thrombus formation $(61,62)$. In patients 
presenting with a CVA, thrombotic manifestations are more frequent than hemorrhage (63). Particularly, those younger than 50 years of age have been reported to present with large-vessel stroke, a feature of macrovascular thrombosis also observed in aHUS and thrombotic thrombocytopenic purpura (64). Clots in the renal vasculature can contribute to acute kidney injury or to myocardial infarction if present in the coronary arteries (65). Another possible link between complement activation and hypercoagulability is that dysfunction of complement regulatory proteins due to underlying genetic alterations may contribute to the pathogenic mechanisms of COVID-19, as suggested in a preprint by Ramlall et al. (44). Such impaired complement regulation could be implicated in both micro- and macrovascular thrombotic events, such as stroke, in addition to primary or secondary TMA (66). In sum, as underlying pathogenic mechanisms are better elucidated, therapeutics can be more appropriately timed and targeted to the injury state (i.e., endothelial injury, hypercoagulability, or complement activation).

\section{Mechanisms of complement-mediated injury in COVID-19}

NETs. Neutrophils and complement are key sentinels of innate immunity and act hand-in-hand to defend the host against invading pathogens and to maintain homeostasis (67). For example, complement opsonization aids in NET generation, while blockade of complement receptor 1 (CR1) and complement receptor 3 (CR3) inhibits NETosis (a type of programmed cell death) in response to certain pathogens. The ability of a pathogen to induce NETosis might therefore inversely correlate with its ability to evade complement activation and opsonization. A pathogen that can prevent complement deposition is probably a less efficient NET inducer (67).

In the case of SARS-CoV-2, an exaggerated host response in patients with COVID-19 centers around the aberrant activation of neutrophils particularly in the lung $(68,69)$. Autopsy reports from those with COVID-19 demonstrated neutrophil infiltration in pulmonary capillaries, acute capillaritis with fibrin deposition, extravasation of neutrophils into the alveolar space, and neutrophilic mucositis (69). Neutrophilia predicts poor outcomes in patients with COVID-19, and the neutrophil-to-lymphocyte ratio is an independent risk factor for severe disease (70). Furthermore, elevated levels of NET-specific markers myeloperoxidase DNA and citrullinated histone H3 were observed in the sera of patients with COVID-19 (69).

Activated neutrophils and NETs contain C3, factor B, and properdin (67). These components are needed to generate and stabilize the AP C3 convertase, thereby amplifying the cascade. The biological relevance of complement activation on NETs is also speculated to facilitate the formation of the anaphylatoxins, C3a and $\mathrm{C} 5 \mathrm{a}$, which further induce recruitment and activation of neutrophils, monocytes, and eosinophils as well as the production of proinflammatory cytokines. Thus, although NETs are beneficial in the host defense against pathogens, sustained NET formation, as seen in COVID-19, can trigger a cascade of inflammatory reactions that damage and destroy surrounding tissues, despite the lack of immune cell infiltration.

Complement and neutrophil activation also work in concert to drive severe COVID-19 manifestations by creating a coagulopathic milieu and therein generating microthrombi, a process which leads to acute lung, kidney, and cardiac injury, as described above and as elaborated on below (67). Complement activation, in conjunction with neutrophilia and dysregulated NET formation, is linked to ARDS, pulmonary inflammation, and thrombotic events, resulting in permanent multiorgan damage. NETs initiate arterial and venous thrombosis by activating the contact pathway of coagulation and enhancing other prothrombotic pathways, resulting in excessive thrombin generation and subsequent C5a generation (67). Thus, we speculate that there is a feedback loop whereby complement activation induces NETosis, which in turn leads to procoagulant activity (e.g., that of thrombin) and ongoing complement activation to further enhance NET formation (Figure 2).

Complement activation and hypercoagulability. The question as to whether complement activation is driving the features of hypercoagulability and microthromboses in COVID-19 is currently an area of immense interest (53). The reasons for the association between the complement and coagulation systems include an evolutionary history that indicates a common origin and recent research revealing considerable crosstalk between the 2 biological processes. The mutual engagement of both systems has been particularly recognized in paroxysmal nocturnal hemoglobinuria (PNH), aHUS, and the antiphospholipid syndrome (71). For example, it has long been known that thrombin can activate C5 in the absence of C3 via a convertase-independent mechanism (72). Similarly, vWF has a complex role in coagulation and reciprocally regulates complement, depending on multimer size (73). Small vWF multimers potentiate the actions of the complement inhibitor factor I, facilitating C3b inactivation, while ultra-large vWF multimers, as observed after tissue injury, provide a binding platform for $\mathrm{C} 3 \mathrm{~b}$ to trigger complement activation via the $\mathrm{AP}(74,75)$. Factor XII cleaves $\mathrm{C} 1 \mathrm{~s}$ and 


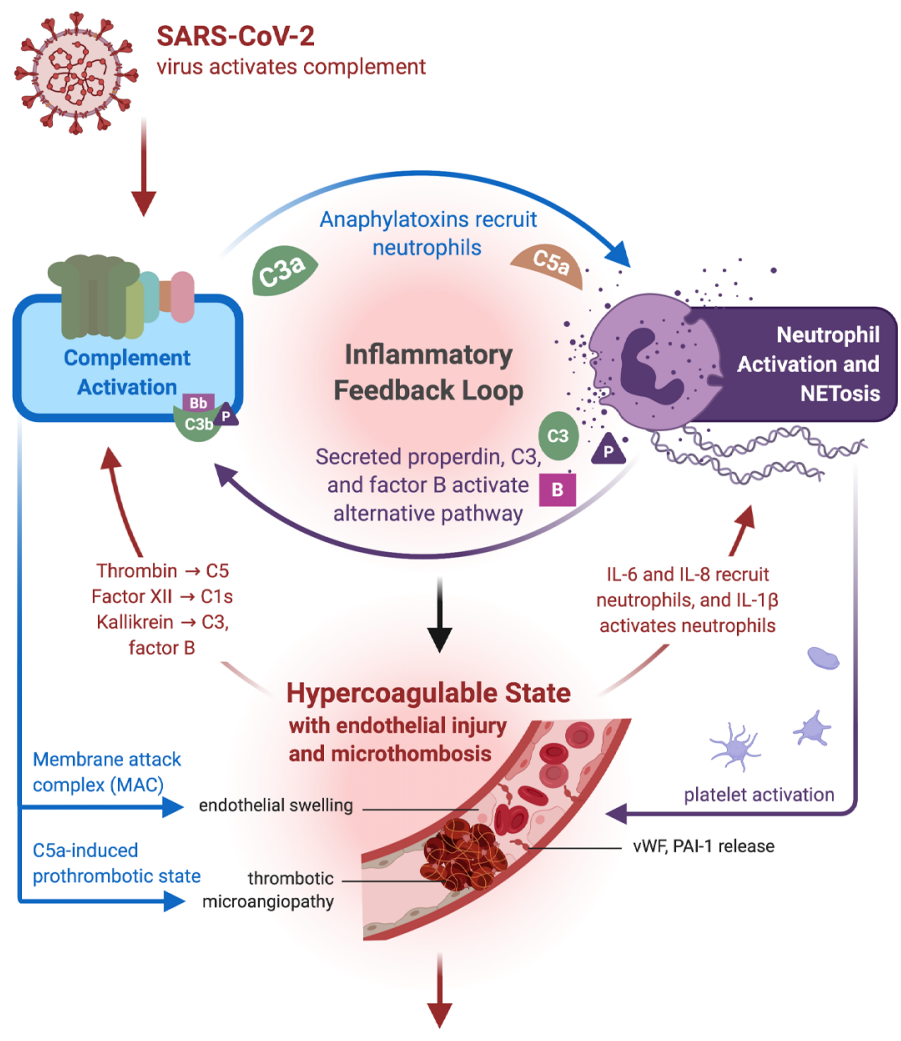

Tissue Injury and Damage

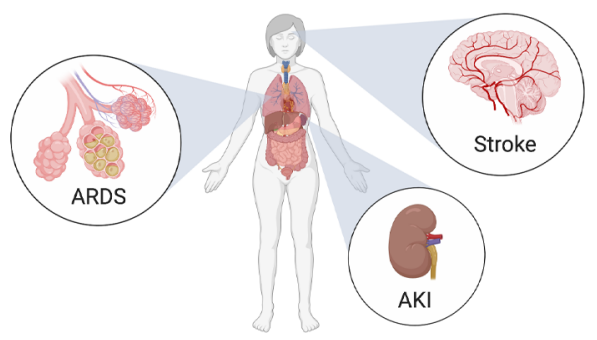

Figure 2. A summary of SARS-CoV-2 and complement activation leading to immune hyperinflammatory reactions and resulting in human pathology. Complement activation generates the proinflammatory polypeptides, C3a and C5a, and recruits neutrophils as well as monocytes. Activated neutrophils generate web-like extracellular traps (NETs), in a process known as NETosis, that contain components such as $C 3$, properdin $(P)$, and factor $B(B)$ that activate the alternative complement pathway and engage an inflammatory feedback loop. Although NETs assist in host defense against pathogens, a sustained response, such as that seen in COVID-19, may incite ongoing inflammation and a hypercoagulable state. Additionally, the membrane attack complex (MAC) also induces endothelial inflammation and tissue injury, leading to the generation of IL- 6 and IL- $1 \beta$, which continue to propagate NETosis. Endothelial injury leads to the generation of vWF multimers. Excess ultralong VWF stabilizes factor VIII activity and prevents the binding of factor I. Endothelial damage also results in the release of plasminogen activator inhibitor-1 (PAI-1), which exacerbates thrombosis, along with C5a-induced release of tissue factor and other prothrombotic proteins. These changes then can augment a complement-coagulation pathway crosstalk, due to serine proteases, such as thrombin and kallikrein, activating the complement system in a convertase-independent manner. Such interactions among endothelial injury, hypercoagulability, and complement activation cause tissue damage, such as acute respiratory distress syndrome (ARDS), acute kidney injury (AKI), and stroke, and are often associated with a thrombotic microangiopathy.

thereby activates the classical complement pathway (76), while kallikrein cleaves both C3 and factor B (77). Platelets themselves may facilitate as well as amplify complement activation $(78,79)$.

Furthermore, C5a exerts a prothrombotic effect by upregulating tissue factor and PAI-1 expression by endothelial cells and monocytes $(80,81)$. The disruption of endothelial cells and platelets by the MAC provides a nidus for prothrombinase assembly (82), and MASPs (initiators of the lectin pathway) have been shown to cleave prothrombin to thrombin (83). Interestingly, complement activation can both facilitate coagulation and interfere with anticoagulation. For example, heparan sulfate is a proteoglycan on endothelial cells that dampens local inflammation and inhibits coagulation. However, endothelial injury is associated with shedding of heparan sulfate via both complement-dependent (exposure to C5a) and -independent mechanisms $(84,85)$.

In addition to these changes that both enhance clot formation and impair the clearance of fibrin, complement activation may also contribute to increased vascular bed resistance in end organs. For example, a current hypothesis is that angiotensin II levels are locally elevated in critically ill patients with severe COVID-19, at least in part, due to downregulation of ACE2 on different cell surfaces (86). In preclinical models of angiotensin II-associated vasculopathy, locally elevated C-reactive protein and TNF- $\alpha$ levels induced C3 expression and were associated with complement activation (defined by elevated C1q, C3, C3c and C5b-9) in the vessel media (87). Thus, such mechanisms may create an amplifying loop in vascular beds wherein endothelial injury and complement activation further accentuate the prothrombotic state. Initial reports also suggest that, in addition to elevated fibrin degradation products (e.g., D-dimer), patients with severe COVID-19 have elevated fibrinogen levels, factor VIII activity, and vWF levels (88-90). 
Platelet count, aPTT, and, occasionally, even PT have been reported as normal or mildly elevated in these patients. Although many of the coagulation components remain to be systematically tested in patients with COVID-19, the currently reported parameters, along with the clinical features, have led to the hypothesis that the coagulation disturbances seen in severe COVID-19 represent a pattern of complement-mediated TMA, rather than DIC $(13,53)$. Thus, in COVID-19, the crosstalk between the complement and coagulation cascades creates a prothrombotic environment associated with adverse outcomes.

Hyperinflammation and cytokine storm. A hyperinflammatory state is observed in patients with severe COVID-19, despite the apparent absence of tissue inflammation outside of the lungs. In a study of 41 hospitalized patients, high plasma levels of proinflammatory cytokines (IL-2, IL-6, IL-7, IL-10, G-CSF, IP-10, MCP-1, MIP-1 $\alpha$, and TNF- $\alpha$ ) were associated with adverse clinical outcomes, such as ARDS, shock, organ failure, and death (91). Although multiple viral pathogens are known to induce this hyperinflammatory state (92), it is unclear how SARS-CoV-2 infection promotes the so-called "cytokine storm."

Complement is well known for promoting immune cell activation and proinflammatory states. Anaphylatoxins $\mathrm{C} 3 \mathrm{a}$ and $\mathrm{C} 5 \mathrm{a}$ are capable of activating neutrophils, mast cells, monocytes/macrophages, basophils, eosinophils, T cells, and B cells (93). This drives a potent proinflammatory response, especially by macrophages and neutrophils, including the expression of TNF- $\alpha$, IL-1 $\beta$, and IL-6 (93). As previously noted, elevated plasma C5a was observed in patients with moderate and severe COVID-19, along with elevated IL-6 (40). Furthermore, upregulation of IL-6 can be a direct sequela of enhanced endothelial cell expression of this cytokine due to mannan-binding lectin pathway activation. Thus, while the hyperin flammatory state observed in COVID-19 likely involves numerous players, these observations suggest that complement plays an important role.

Complement genes, including those encoding $\mathrm{C} 1 \mathrm{q}, \mathrm{C} 2$, factor $\mathrm{B}$, and factor $\mathrm{D}$, are also part of the IFN-stimulated gene response (94). Elevated IFN levels and undesirable engagement of complement have been noted in patients with posttransplant TMA (95), suggesting an IFN-complement loop that contributes to a hypercoagulable state. However, SARS-CoV-2 induces an abbreviated IFN-stimulated gene signature with low systemic IFN levels (96) that likely serves as a viral evasion strategy. Therefore, how the attenuated IFN response influences complement levels and activity in COVID-19 remains to be determined. Does the early attenuated IFN response reduce complement component expression, preventing sufficient complement activation to assist in viral clearance early in disease? Does the active viral replication later result in hyperproduction of IFN and influx of neutrophils and macrophages, leading to a hyperinflammatory state that drives an overexuberant complement response, contributing to severe manifestations, such as ARDS? Testing these intriguing hypotheses will shed additional insight into the crosstalk between these systems and will also help to determine the efficacy and timing of IFN administration, which is key to yielding a protective response.

\section{Intracellular complement viral defense: the complosome}

Traditionally, complement has been considered primarily a serum-centric system in which complement activation occurs only in the extracellular milieu. Thus, opsonization of viruses would occur outside the cell. However, recent studies reveal that complement also has a rich and robust intracellular arsenal of components, the complosome, that provides immune defense as well as mediates key interactions for host cell functioning (reviewed in refs. 19, 97). Further, proteins such as C3 and C5 can be cleaved in a noncanonical manner inside the cell $(98,99)$. These findings suggest that virus opsonization, especially by C3b, may occur intracellularly as well. For example, C3 plays a role in detecting and disabling intracellular pathogens (25). Tagging the virus with $\mathrm{C} 3 \mathrm{~b} / \mathrm{iC} 3 \mathrm{~b}$ and then infecting epithelial cells activated cytoplasmic sensors of $\mathrm{C} 3$ and the mitochondrial antiviral signaling pathway. This in turn led to potent responses by NF- $\mathrm{B}$, activating protein 1, and the IFN regulatory factor transcriptional pathways. These data indicate that intracellular C3 fragments serve as damage-associated molecular patterns to enhance intracellular innate immunity.

While elucidation of the complosome has centered primarily on T cells (20), the intracellular complement system likely functions in most, if not all, cells $(19,97)$. Defining if and how complement activation modulates these responses in the context of SARS-CoV-2 remains to be identified.

However, a major consideration in how intracellular complement modulates cellular functions is the source of these intracellular proteins. We have previously demonstrated that cells internalize C3 in the form of $\mathrm{C} 3\left(\mathrm{H}_{2} \mathrm{O}\right)$ from the extracellular space, and this internalization increases the production of IL-6 and IL-17 from effector T cells (100). Thus, this rapid internalization of C3 is likely to alter the proinflammatory responses when an exogenous source of C3 is made available (e.g., in the context of barrier disruption 
and associated plasma leak in acute lung injury) and accelerated turnover from $\mathrm{C} 3$ to $\mathrm{C} 3\left(\mathrm{H}_{2} \mathrm{O}\right)$ in the context of ongoing inflammation (101). The recent discovery that intracellular C3 protects human airway epithelial cells (AECs) from stress-associated death may also have implications for COVID-19 (102). In this study, AECs were unique compared with other cell types in containing large intracellular stores of de novo-synthesized C3. Proinflammatory cytokine exposure increased both stored and secreted forms of C3. Additionally, AECs took up C3 from exogenous sources that then mitigated stress-associated cellular death. These studies suggest that modulation of intracellular C3 in AECs may have beneficial effects. Thus, approaches that inhibit C3 may need to consider its intracellular permeability and effects on cellular viability. Elucidating the effects of both intracellular and extracellular key complement components, such as C3 and $\mathrm{C} 5$, could provide a greater understanding of how viruses regulate complement activation and thereby suggest appropriate therapeutic targeting strategies.

\section{Targeting complement: current and future trials}

Multiple therapeutic agents targeting complement activation are currently being studied for COVID-19 (Figure 1). The most commonly used complement inhibitor in clinical practice currently is eculizumab, a humanized monoclonal antibody to C5. Eculizumab is FDA-approved for patients with PNH, aHUS, myasthenia gravis, and neuromyelitis optica spectrum disorder (NMOSD) (103). A case series of patients with COVID-19 receiving eculizumab (104) suggests that the drug remains well-tolerated in these patients. However, additional multicentric data, along with a better understanding of how blocking complement affects critically ill patients, are urgently needed. As a result, there are 2 ongoing trials of eculizumab in COVID-19 in Europe (NCT04355494, an open-label, multicenter, expanded access program, and NCT04346797, CORIMUNO19-ECU, a phase II trial and part of a larger CORIMUNO19 study). While most of these studies primarily focus on adult patients with COVID-19 with severe pneumonia, acute lung injury, or ARDS requiring oxygen supplementation, some patients also have features overlapping with aHUS. These cases prompt consideration as to whether most patients would benefit from this therapy versus a subset of those who have microangiopathy-like features (schistocytes, microthromboses [with end-organ damage], and other laboratory evidence of complement activation). Importantly, identifying the correct timing to initiate this treatment will likely be a critical issue.

There are ongoing clinical trials examining ravulizumab (phase III, NCT04369469), IFX-1 (phase II and III, NCT04333420), avdoralimab (phase II, NCT04371367), and AMY-101 (compassionate use). Ravulizum$\mathrm{ab}$, like eculizumab, is a recombinant humanized anti-C5 monoclonal antibody that has a prolonged half-life compared with eculizumab (105). Ravulizumab is FDA approved for use in aHUS and PNH, and its efficacy and safety are currently being evaluated in acute lung injury and cytokine storm associated with COVID-19.

IFX-1 is a monoclonal antibody that blocks the effect of C5a and, therefore, keeps the formation of the MAC intact by not interfering with $\mathrm{C} 5 \mathrm{~b}$ generation or function (106). It has shown efficacy in nonhuman primate models of toxin- and virus-induced acute lung injury (107, 108). Avdoralimab (IPH5401)

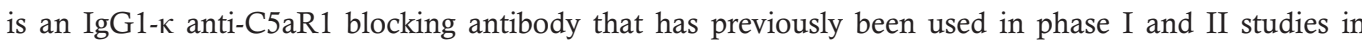
combination with checkpoint inhibitors for solid tumors (NCT03665129) and is being studied in patients with COVID-19 (NCT04371367) who need $\geq 5 \mathrm{~L} / \mathrm{min}$ supplemental oxygen to maintain oxygen saturation (SpO2) $>93 \%$ or those with ARDS, who need invasive mechanical ventilation with a $\mathrm{PaO} 2 / \mathrm{FiO} 2$ ratio $<300$ for more than 24 hours. A newer class of C3 inhibitors is being tested for COVID-19-associated ARDS (109), with safety studies having been conducted in nonhuman primates (110). Another consideration is the use of the MASP-2 inhibitor narsoplimab (OMS721), given the interaction between MASP-2 and the N-protein of SARS-CoV-2 (27).

An especially important consideration for any trial of complement inhibition is the predisposition to infection by encapsulated organisms. This becomes even more important in critically ill patients, including those with COVID-19, many of whom may not have received meningococcal vaccination in the past. Despite this major concern, the safety of eculizumab has been demonstrated in postmarketing surveillance $(111,112)$. Nevertheless, a tailored approach for careful patient selection, antibiotic prophylaxis during and potentially for a period of time after treatment cessation, will be necessary to minimize any unintended adverse effects associated with infections. Thus, while complement activation has a role in viral opsonization, a deeper understanding of the dynamics of altering viral proliferation versus the hyperimmune response will help to determine the appropriate time to introduce these treatments. 


\section{Conclusion}

Multiple features of severe SARS-CoV-2 infection suggest that complement activation plays a critical role in the pathogenesis of COVID-19, particularly during exaggerated immune responses. However, the variable clinical course and the ability of this system to modulate a viral infection is obviously a serious concern. Therefore, deciding at what point complement intervention is appropriate is a key and an unanswered issue. For example, one could easily envision that complement inhibition (of 1 or all 3 major pathways) could prove deleterious in the first week, as during this time complement serves as a "friend." On the other hand, in the second or third weeks of infection, complement intervention could prove critical, since at this time it becomes a "foe." Hopefully, informative clinical trials on these and other key issues will provide much-needed answers.

\section{Acknowledgments}

The authors would like to thank Ben Palanca, Kristen Sanfilippo, and Anthony Chang for informative discussions that helped to shape the manuscript. Figures were partially created using BioRender. AJ is supported by the Barnes Jewish Hospital Foundation Fund and is a coinvestigator on a multicenter study funded by Alexion (NCT04369469). JPA is supported by NIH/National Institute of General Medical Sciences (R35-GM136352-01 and 2R01-GM99111-23). AHJK is supported by NIH/National Institute of Arthritis and Musculoskeletal and Skin Diseases (P30-AR073752) and the Rheumatology Research Foundation. HSK is funded by the Children's Discovery Institute of Washington University and St. Louis Children's Hospital (PD-FR-2020-867), the NIH (K08-HL148510), and the American Lung Association (RG-575308). HSK is currently a principal investigator on a multicenter study funded by Alexion on the use of complement inhibitors in severe COVID-19 (NCT04369469). The content is solely the responsibility of the authors and does not necessarily represent the official views of the NIH.

Address correspondence to: Alfred H.J. Kim, Department of Medicine, Division of Rheumatology, Washington University School of Medicine, 660 S. Euclid Avenue, Campus Box 8045, Saint Louis, Missouri 63110, USA. Phone: 314.362.4785; Email: akim@wustl.edu. Or to: Hrishikesh S. Kulkarni, Department of Medicine, Division of Pulmonary and Critical Care Medicine, Washington University School of Medicine, 4523 Clayton Avenue, Campus Box 8052, Saint Louis, Missouri 63110, USA. Phone: 314.273.9008; Email: hkulkarn@wustl.edu.

1. Vabret N, et al. Immunology of COVID-19: Current State of the Science. Immunity. 2020;52(6):910-941.

2. Bloch EM, et al. Deployment of convalescent plasma for the prevention and treatment of COVID-19. J Clin Invest. 2020;130(6):2757-2765.

3. Angus DC, et al. The REMAP-CAP (Randomized Embedded Multifactorial Adaptive Platform for Community-acquired Pneumonia) Study. Rationale and Design. Ann Am Thorac Soc. 2020;17(7):879-891.

4. Coronaviridae Study Group of the International Committee on Taxonomy of Viruses. The species Severe acute respiratory syndrome-related coronavirus: classifying 2019-nCoV and naming it SARS-CoV-2. Nat Microbiol. 2020;5(4):536-544.

5. Henrickson SE. Learning from our immunological history: What can SARS-CoV teach us about SARS-CoV-2? Sci Immunol. 2020;5(46):eabb8618.

6. Guan WJ, et al. Clinical characteristics of coronavirus disease 2019 in China. N Engl J Med. 2020;382(18):1708-1720.

7. Wang D, et al. Clinical characteristics of 138 hospitalized patients with 2019 novel coronavirus-infected pneumonia in Wuhan, China. JAMA. 2020;323(11): 1061-1069.

8. Phua J, et al. Intensive care management of coronavirus disease 2019 (COVID-19): challenges and recommendations. Lancet Respir Med. 2020;8(5):506-517.

9. Xu X, et al. Effective treatment of severe COVID-19 patients with tocilizumab. Proc Natl Acad Sci USA. 2020;117(20):10970-10975

10. Mehta P, et al. COVID-19: consider cytokine storm syndromes and immunosuppression. Lancet. 2020;395(10229):1033-1034.

11. Pedersen SF, Ho YC. SARS-CoV-2: a storm is raging. J Clin Invest. 2020;130(5):2202-2205.

12. Zhang Y, et al. Coagulopathy and antiphospholipid antibodies in patients with Covid-19. N Engl J Med. 2020;382(17):e38.

13. Magro C, et al. Complement associated microvascular injury and thrombosis in the pathogenesis of severe COVID-19 infection: A report of five cases. Transl Res. 2020;220:1-13.

14. Di Mascio D, et al. Outcome of Coronavirus spectrum infections (SARS, MERS, COVID 1 -19) during pregnancy: a systematic review and meta-analysis. Am J Obstet Gynecol MFM. 2020;2(2):100107.

15. Shen B, et al. Proteomic and Metabolomic Characterization of COVID-19 Patient Sera. Cell. 2020;182(1):59-72.e15.

16. Li Y, et al. Urine proteome of COVID-19 patients. medRxiv. https://doi.org/10.1101/2020.05.02.20088666. Published May 6,2020. Accessed June 29, 2020.

17. Risitano AM, et al. Complement as a target in COVID-19? Nat Rev Immunol. 2020;20(6):343-344.

18. Nonaka M, Kimura A. Genomic view of the evolution of the complement system. Immunogenetics. 2006;58(9):701-713.

19. Liszewski MK, Elvington M, Kulkarni HS, Atkinson JP. Complement's hidden arsenal: New insights and novel functions inside 
the cell. Mol Immunol. 2017;84:2-9.

20. West EE, Kolev M, Kemper C. Complement and the regulation of T cell responses. Annu Rev Immunol. 2018;36:309-338.

21. Nasir A, Caetano-Anollés G. A phylogenomic data-driven exploration of viral origins and evolution. Sci Adv. 2015;1(8):e1500527.

22. Agrawal P, Nawadkar R, Ojha H, Kumar J, Sahu A. Complement evasion strategies of viruses: An Overview. Front Microbiol. 2017;8:1117.

23. Stoermer KA, Morrison TE. Complement and viral pathogenesis. Virology. 2011;411(2):362-373.

24. Liszewski MK, Bertram P, Leung MK, Hauhart R, Zhang L, Atkinson JP. Smallpox inhibitor of complement enzymes (SPICE): regulation of complement activation on cells and mechanism of its cellular attachment. J Immunol. 2008;181(6):4199-4207.

25. Tam JC, Bidgood SR, McEwan WA, James LC. Intracellular sensing of complement C3 activates cell autonomous immunity. Science. 2014;345(6201):1256070.

26. Gralinski LE, et al. Complement activation contributes to severe acute respiratory syndrome coronavirus pathogenesis. $m B i o$. 2018;9(5):e01753-18.

27. Gao T, et al. Highly pathogenic coronavirus $\mathrm{N}$ protein aggravates lung injury by MASP-2-mediated complement over-activation. medRxiv. https://doi.org/10.1101/2020.03.29.20041962. Published June 18, 2020. Accessed June 29, 2020.

28. Hernandez AM, Holodick NE. Editorial: Natural antibodies in health and disease. Front Immunol. $2017 ; 8: 1795$.

29. Zhao J, et al. Relationship between the ABO Blood Group and the COVID-19 Susceptibility. medRxiv. https://doi.org/10.1101 /2020.03.11.20031096. Published March 27, 2020. Accessed June 29, 2020

30. Zietz M, Tatonetti NP. Testing the association between blood type and COVID-19 infection, intubation, and death. medRxiv. https://doi.org/10.1101/2020.04.08.20058073. Published April 11, 2020. Accessed June 29, 2020.

31. Ellinghaus D, et al. The ABO blood group locus and a chromosome 3 gene cluster associate with SARS-CoV-2 respiratory failure in an Italian-Spanish genome-wide association analysis. medRxiv. https://doi.org/10.1101/2020.05.31.20114991. Published June 2, 2020. Accessed June 29, 2020.

32. Cheng Y, et al. ABO blood group and susceptibility to severe acute respiratory syndrome. JAMA. 2005;293(12):1450-1451

33. Preece AF, Strahan KM, Devitt J, Yamamoto F, Gustafsson K. Expression of ABO or related antigenic carbohydrates on viral envelopes leads to neutralization in the presence of serum containing specific natural antibodies and complement. Blood. 2002;99(7):2477-2482

34. Guillon P, et al. Inhibition of the interaction between the SARS-CoV spike protein and its cellular receptor by anti-histo-blood group antibodies. Glycobiology. 2008;18(12):1085-1093.

35. Rodriguez-Zhurbenko N, Quach TD, Hopkins TJ, Rothstein TL, Hernandez AM. Human B-1 cells and B-1 cell antibodies change with advancing age. Front Immunol. 2019;10:483.

36. Zhang G, et al. Clinical features and short-term outcomes of 221 patients with COVID-19 in Wuhan, China. J Clin Virol. 2020;127:104364.

37. Onder G, Rezza G, Brusaferro S. Case-fatality rate and characteristics of patients dying in relation to COVID-19 in Italy. JAMA 2020;323(18):1775-1776.

38. Richardson S, et al. Presenting characteristics, comorbidities, and outcomes among 5700 patients hospitalized with COVID-19 in the New York City area. JAMA. 2020;323(20):2052-2059.

39. CDC COVID-19 Response Team. Severe Outcomes Among Patients with Coronavirus Disease 2019 (COVID-19) - United States, February 12-March 16, 2020. MMWR Morb Mortal Wkly Rep. 2020;69(12):343-346.

40. Cugno M, et al. Complement activation in patients with COVID-19: A novel therapeutic target. J Allergy Clin Immunol. 2020;146(1):215-217.

41. Diao B, et al. Human kidney is a target for novel severe acute respiratory syndrome coronavirus 2 (SARS-CoV-2) infection medRxiv. https://doi.org/10.1101/2020.03.04.20031120. Published April 10, 2020. Accessed June 29, 2020.

42. Tang S, Sheerin NS, Zhou W, Brown Z, Sacks SH. Apical proteins stimulate complement synthesis by cultured human proxima tubular epithelial cells. J Am Soc Nephrol. 1999;10(1):69-76.

43. Ichida S, Yuzawa Y, Okada H, Yoshioka K, Matsuo S. Localization of the complement regulatory proteins in the normal human kidney. Kidney Int. 1994;46(1):89-96.

44. Ramlall V, Thangaraj P, Tatonetti NP, Shapira SD. Identification of Immune complement function as a determinant of adverse SARS-CoV-2 infection outcome. medRxiv. https://doi.org/10.1101/2020.05.05.20092452. Published June 6,2020. Accessed June 29, 2020.

45. Liszewski MK, Java A, Schramm EC, Atkinson JP. Complement dysregulation and disease: insights from contemporary genetics. Annu Rev Pathol. 2017;12:25-52.

46. Puelles VG, et al. Multiorgan and renal tropism of SARS-CoV-2 [published online May 13,2020]. N Engl J Med. https://doi. org/10.1056/NEJMc2011400.

47. Terpos E, et al. Hematological findings and complications of COVID-19. Am J Hematol. 2020;95(7):834-847.

48. Varga Z, et al. Endothelial cell infection and endotheliitis in COVID-19. Lancet. 2020;395(10234):1417-1418.

49. Bryce C, et al. Pathophysiology of SARS-CoV-2: targeting of endothelial cells renders a complex disease with thrombotic microangiopathy and aberrant immune response. The Mount Sinai COVID-19 autopsy experience. medRxiv. https://doi.org/1 0.1101/2020.05.18.20099960. Published May 22, 2020. Accessed June 29, 2020.

50. Su H, et al. Renal histopathological analysis of 26 postmortem findings of patients with COVID-19 in China. Kidney Int 2020;98(1):219-227.

51. Jhaveri KD, et al. Thrombotic microangiopathy in a patient with COVID-19. Kidney Int. 2020;98(2):509-512.

52. Timmermans SAMEG, et al. C5b9 formation on endothelial cells reflects complement defects among patients with renal thrombotic microangiopathy and severe hypertension. J Am Soc Nephrol. 2018;29(8):2234-2243.

53. Gavriilaki E, Brodsky RA. Severe COVID-19 infection and thrombotic microangiopathy: success does not come easily. $\mathrm{Br} J \mathrm{Hae}-$ matol. 2020;189(6):e227-e230

54. Escher R, Breakey N, Lämmle B. Severe COVID-19 infection associated with endothelial activation. Thromb Res. 2020;190:62

55. Panigada M, et al. Hypercoagulability of COVID-19 patients in intensive care unit: A report of thromboelastography findings 
and other parameters of hemostasis. J Thromb Haemost. 2020;18(7):1738-1742.

56. Folco EJ, et al. Neutrophil extracellular traps induce endothelial cell activation and tissue factor production through interleukin-1 $\alpha$ and cathepsin G. Arterioscler Thromb Vasc Biol. 2018;38(8):1901-1912.

57. Michiels C, Arnould T, Remacle J. Endothelial cell responses to hypoxia: initiation of a cascade of cellular interactions. Biochim Biophys Acta. 2000;1497(1):1-10.

58. Zhou F, et al. Clinical course and risk factors for mortality of adult inpatients with COVID-19 in Wuhan, China: a retrospective cohort study. Lancet. 2020;395(10229):1054-1062.

59. Marini JJ, Gattinoni L. Management of COVID-19 respiratory distress. JAMA. 2020;323(22):2329-2330.

60. Fox SE, Akmatbekov A, Harbert JL, Li G, Quincy Brown J, Vander Heide RS. Pulmonary and cardiac pathology in African American patients with COVID-19: an autopsy series from New Orleans. Lancet Respir Med. 2020;8(7):681-686

61. Ackermann M, et al. Pulmonary Vascular Endothelialitis, Thrombosis, and Angiogenesis in Covid-19. N Engl J Med. 2020;383(2):120-128.

62. Schaller T, et al. Postmortem examination of patients with COVID-19. JAMA. 2020;323(24):2518-2520.

63. Mao L, et al. Neurologic manifestations of hospitalized patients with coronavirus disease 2019 in Wuhan, China. JAMA Neurol. 2020;77(6):683-690.

64. Oxley TJ, et al. Large-vessel stroke as a presenting feature of Covid-19 in the young. N Engl J Med. 2020;382(20):e60.

65. Batlle D, et al. Acute Kidney Injury in COVID-19: Emerging Evidence of a Distinct Pathophysiology. J Am Soc Nephrol. 2020;31(7):1380-1383.

66. Ueda Y, et al. Murine systemic thrombophilia and hemolytic uremic syndrome from a factor H point mutation. Blood. 2017;129(9):1184-1196.

67. de Bont CM, Boelens WC, Pruijn GJM. NETosis, complement, and coagulation: a triangular relationship. Cell Mol Immunol. 2019;16(1):19-27.

68. Barnes BJ, et al. Targeting potential drivers of COVID-19: Neutrophil extracellular traps. J Exp Med. 2020;217(6):e20200652

69. Zuo Y, et al. Neutrophil extracellular traps in COVID-19. JCI Insight. 2020;5(11):138999.

70. Liu J, et al. Neutrophil-to-lymphocyte ratio predicts severe illness patients with 2019 novel coronavirus in the early stage. medRxiv. https://doi.org/10.1101/2020.02.10.20021584. Published February 12, 2020. Accessed June 29, 2020.

71. Dzik S. Complement and coagulation: cross talk through time. Transfus Med Rev. 2019;33(4):199-206.

72. Huber-Lang M, et al. Generation of C5a in the absence of C3: a new complement activation pathway. Nat Med. 2006;12(6):682-687.

73. Hattori R, Hamilton KK, McEver RP, Sims PJ. Complement proteins C5b-9 induce secretion of high molecular weight multimers of endothelial von Willebrand factor and translocation of granule membrane protein GMP-140 to the cell surface. $J$ Biol Chem. 1989;264(15):9053-9060.

74. Feng S, Liang X, Kroll MH, Chung DW, Afshar-Kharghan V. von Willebrand factor is a cofactor in complement regulation. Blood. 2015;125(6):1034-1037.

75. Bettoni S, et al. Interaction between multimeric von Willebrand factor and complement: a fresh look to the pathophysiology of microvascular thrombosis. J Immunol. 2017;199(3):1021-1040.

76. Ghebrehiwet B, Randazzo BP, Dunn JT, Silverberg M, Kaplan AP. Mechanisms of activation of the classical pathway of complement by Hageman factor fragment. J Clin Invest. 1983;71(5):1450-1456.

77. Irmscher S, et al. Kallikrein cleaves C3 and activates complement. J Innate Immun. 2018;10(2):94-105.

78. Peerschke EI, Yin W, Ghebrehiwet B. Platelet mediated complement activation. Adv Exp Med Biol. 2008;632:81-91.

79. Subramaniam S, et al. Distinct contributions of complement factors to platelet activation and fibrin formation in venous thrombus development. Blood. 2017;129(16):2291-2302.

80. Ritis K, et al. A novel C5a receptor-tissue factor cross-talk in neutrophils links innate immunity to coagulation pathways. $J$ Immunol. 2006;177(7):4794-4802.

81. Foley JH, Conway EM. Cross Talk Pathways Between Coagulation and Inflammation. Circ Res. 2016;118(9):1392-1408.

82. Wiedmer T, Esmon CT, Sims PJ. Complement proteins C5b-9 stimulate procoagulant activity through platelet prothrombinase. Blood. 1986;68(4):875-880.

83. Krarup A, Wallis R, Presanis JS, Gál P, Sim RB. Simultaneous activation of complement and coagulation by MBL-associated serine protease 2. PLoS One. 2007;2(7):e623.

84. Platt JL, Dalmasso AP, Lindman BJ, Ihrcke NS, Bach FH. The role of C5a and antibody in the release of heparan sulfate from endothelial cells. Eur J Immunol. 1991;21(11):2887-2890.

85. Collins LE, Troeberg L. Heparan sulfate as a regulator of inflammation and immunity. J Leukoc Biol. 2019;105(1):81-92.

86. Glowacka I, et al. Differential downregulation of ACE2 by the spike proteins of severe acute respiratory syndrome coronavirus and human coronavirus NL63. J Virol. 2010;84(2):1198-1205.

87. Shagdarsuren E, et al. Complement activation in angiotensin II-induced organ damage. Circ Res. 2005;97(7):716-724.

88. Ranucci M, et al. The procoagulant pattern of patients with COVID-19 acute respiratory distress syndrome. J Thromb Haemost. 2020;18(7):1747-1751

89. Helms J, et al. High risk of thrombosis in patients with severe SARS-CoV-2 infection: a multicenter prospective cohort study. Intensive Care Med. 2020;46(6):1089-1098.

90. Xiong M, Liang X, Wei YD. Changes in blood coagulation in patients with severe coronavirus disease 2019 (COVID-19): a meta-analysis. Br J Haematol. 2020;189(6):1050-1052.

91. Huang C, et al. Clinical features of patients infected with 2019 novel coronavirus in Wuhan, China. Lancet. 2020;395(10223):497-506.

92. Pinto AK, et al. Deficient IFN signaling by myeloid cells leads to MAVS-dependent virus-induced sepsis. PLoS Pathog 2014;10(4):e1004086.

93. Markiewski MM, Lambris JD. The role of complement in inflammatory diseases from behind the scenes into the spotlight. Am J Pathol. 2007;171(3):715-727.

94. Ripoche J, et al. Interferon gamma induces synthesis of complement alternative pathway proteins by human endothelial cells in culture. J Exp Med. 1988;168(5):1917-1922. 
95. Jodele S, et al. Interferon-complement loop in transplant-associated thrombotic microangiopathy. Blood Adv. 2020;4(6):1166-1177. 96. Blanco-Melo D, et al. Imbalanced host response to SARS-CoV-2 drives development of COVID-19. Cell. 2020;181(5):1036-1045.e9.

97. Arbore G, Kemper C, Kolev M. Intracellular complement - the complosome - in immune cell regulation. Mol Immunol. 2017;89:2-9.

98. Liszewski MK, et al. Intracellular complement activation sustains T cell homeostasis and mediates effector differentiation. Immunity. 2013;39(6):1143-1157.

99. Arbore G, et al. T helper 1 immunity requires complement-driven NLRP3 inflammasome activity in CD4+ T cells. Science. 2016;352(6292):aad1210.

100. Elvington M, Liszewski MK, Bertram P, Kulkarni HS, Atkinson JP. A C3(H20) recycling pathway is a component of the intracellular complement system. J Clin Invest. 2017;127(3):970-981.

101. Elvington M, et al. Development and optimization of an ELISA to quantitate $\mathrm{C} 3(\mathrm{H} 2 \mathrm{O})$ as a marker of human disease. Front Immunol. 2019;10:703.

102. Kulkarni HS, et al. Intracellular C3 protects human airway epithelial cells from stress-associated cell death. Am J Respir Cell Mol Biol. 2019;60(2):144-157.

103. Zelek WM, Xie L, Morgan BP, Harris CL. Compendium of current complement therapeutics. Mol Immunol. 2019;114:341-352.

104. Diurno F, et al. Eculizumab treatment in patients with COVID-19: preliminary results from real life ASL Napoli 2 Nord experience. Eur Rev Med Pharmacol Sci. 2020;24(7):4040-4047.

105. Lee JW, et al. Ravulizumab (ALXN1210) vs eculizumab in adult patients with PNH naive to complement inhibitors: the 301 study. Blood. 2019;133(6):530-539.

106. Riedemann NC, et al. Controlling the anaphylatoxin C5a in diseases requires a specifically targeted inhibition. Clin Immunol. 2017; 180:25-32

107. Sun S, et al. Treatment with anti-C5a antibody improves the outcome of H7N9 virus infection in African green monkeys. Clin Infect Dis. 2015;60(4):586-595.

108. Sun S, et al. Treatment of paraquat-induced lung injury with an anti-C5a antibody: potential clinical application. Crit Care Med. 2018;46(5):e419-e425

109. Mastaglio S, et al. The first case of COVID-19 treated with the complement C3 inhibitor AMY-101. Clin Immunol. 2020;215:108450.

110. Reis ES, et al. Safety profile after prolonged C3 inhibition. Clin Immunol. 2018;197:96-106.

111. Kato H, et al. Safety and effectiveness of eculizumab for adult patients with atypical hemolytic-uremic syndrome in Japan: interim analysis of post-marketing surveillance. Clin Exp Nephrol. 2019;23(1):65-75.

112. Socié G, et al. Eculizumab in paroxysmal nocturnal haemoglobinuria and atypical haemolytic uraemic syndrome: 10-year pharmacovigilance analysis. Br J Haematol. 2019;185(2):297-310. 